OPEN ACCESS

Edited by:

Andrea Gomez-Zavaglia, Centro de Investigación y Desarrollo en Criotecnología de Alimentos CONICET, Argentina

Reviewed by: Yong Liu,

Hunan Plant Protection Institute,

China

Katri Johanna Björkroth, University of Helsinki, Finland

*Correspondence: Séamus Fanning sfanning@ucd.ie

Specialty section:

This article was submitted to

Food Microbiology,

a section of the journal

Frontiers in Microbiology

Received: 30 May 2016 Accepted: 20 October 2016 Published: 11 November 2016

Citation:

Yu Z, Gunn L, Brennan E, Reid R, Wall PG, Gaora PÓ, Hurley D, Bolton D and Fanning S (2016)

Complete Genome Sequence of Clostridium estertheticum DSM 8809, a Microbe Identified in Spoiled Vacuum Packed Beef

Front. Microbiol. 7:1764. doi: 10.3389/fmicb.2016.01764

\section{Complete Genome Sequence of Clostridium estertheticum DSM 8809, a Microbe Identified in Spoiled Vacuum Packed Beef}

\author{
Zhongyi Yu', Lynda Gunn', Evan Brennan', Rachael Reid², Patrick G. Wall'1, \\ Peadar Ó. Gaora', Daniel Hurley ${ }^{1}$, Declan Bolton² and Séamus Fanning ${ }^{1 *}$ \\ 1 UCD-Centre for Food Safety, School of Public Health, Physiotherapy and Sports Science, School of Biomedical and \\ Biomolecular Science, University College Dublin, Dublin, Ireland, ${ }^{2}$ Teagasc Food Research Centre, Dublin, Ireland
}

Blown pack spoilage (BPS) is a major issue for the beef industry. Etiological agents of BPS involve members of a group of Clostridium species, including Clostridium estertheticum which has the ability to produce gas, mostly carbon dioxide, under anaerobic psychotrophic growth conditions. This spore-forming bacterium grows slowly under laboratory conditions, and it can take up to 3 months to produce a workable culture. These characteristics have limited the study of this commercially challenging bacterium. Consequently information on this bacterium is limited and no effective controls are currently available to confidently detect and manage this production risk. In this study the complete genome of C. estertheticum DSM 8809 was determined by SMRT ${ }^{\circledR}$ sequencing. The genome consists of a circular chromosome of $4.7 \mathrm{Mbp}$ along with a single plasmid carrying a potential tellurite resistance gene tehB and a Tn3like resolvase-encoding gene tnpR. The genome sequence was searched for central metabolic pathways that would support its biochemical profile and several enzymes contributing to this phenotype were identified. Several putative antibiotic/biocide/metal resistance-encoding genes and virulence factors were also identified in the genome, a feature that requires further research. The availability of the genome sequence will provide a basic blueprint from which to develop valuable biomarkers that could support and improve the detection and control of this bacterium along the beef production chain.

Keywords: blown pack spoilage, Clostridium estertheticum, vacuum packed beef, whole genome sequencing, food quality

\section{INTRODUCTION}

Clostridium estertheticum is a Gram-positive, spore-forming bacterium that is recognized as a causative agent of blown pack spoilage (BPS) in vacuum packed beef products (Bolton et al., 2015). Such events, which may occur as soon as 4 weeks following storage, can lead to economic losses for a meat producer. Despite the implementation of good manufacturing practice (GMP), BPS, including the production of carbon dioxide along with a metallic sheen on the meat, may occur and meat spoiled in this way has no commercial value (Broda et al., 1996). 


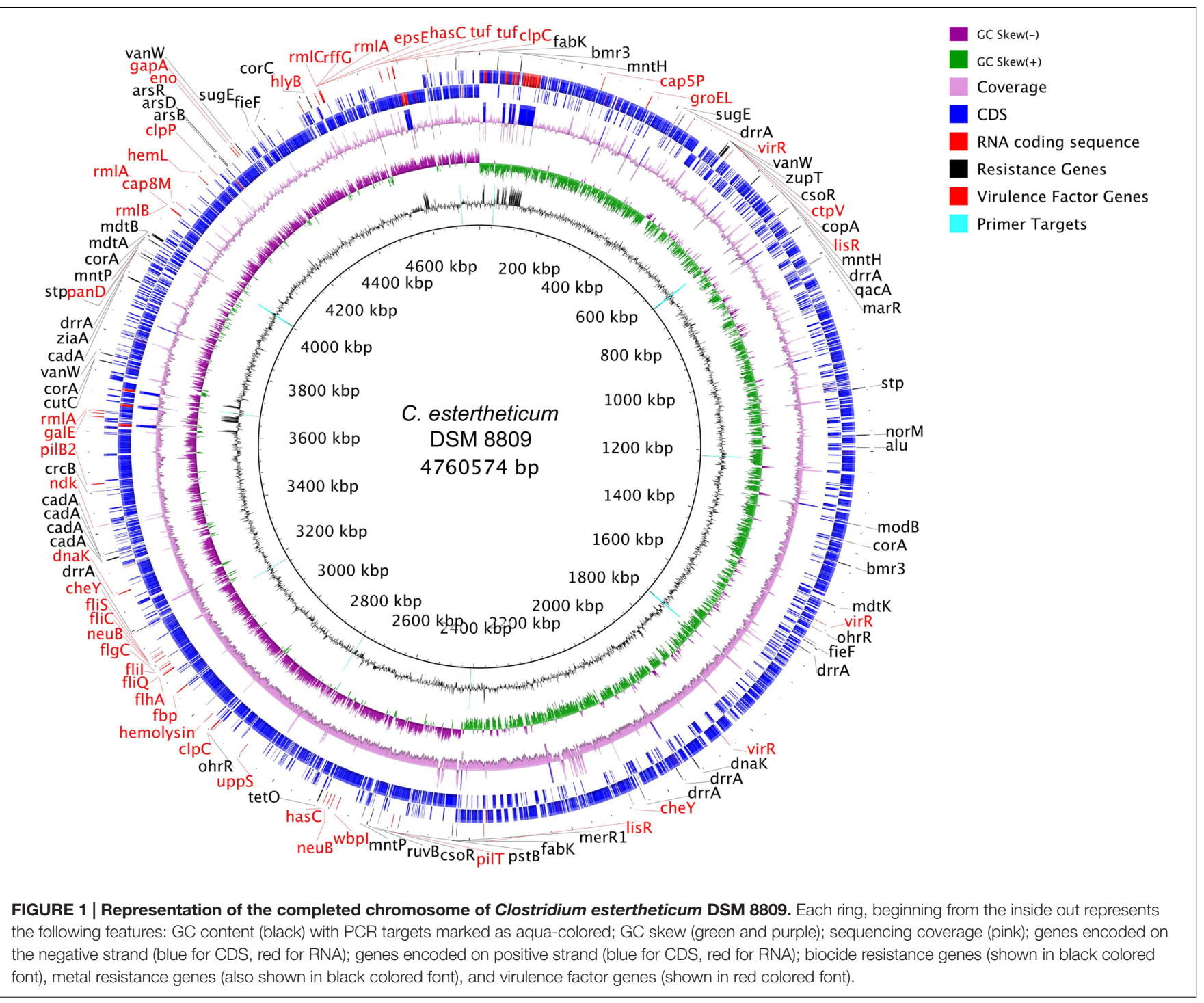

Besides the associated economic loss, controlling BPS continues to be challenging. One strategy deployed is to decontaminate the abattoir with peroxyacetic acid, a known corrosive chemical treatment that exerts a negative impact on the fabric of the production site. Despite these cleaning protocols, spores can remain unaffected, only to germinate at a later stage. The potential occurrence of BPS is one of the main reasons that beef abattoirs cannot adopt the hot/warm boning technique, a measure that would support improvements in beef quality, at a reduced cost through better production methods (Bolton et al., 2015).

Several bacterial species of the genus Clostridium have been recognized in BPS episodes. These include C. estertheticum, Clostridium gasigenes, and Clostridium ruminantium, all of which can be difficult to culture and study in the bacteriology laboratory. Several weeks or in some cases even months are necessary to obtain a workable culture. Thus, even the most routine laboratory protocols can be frustrating to implement and to troubleshoot. Phenotype-based approaches for the detection of these bacteria are not a feasible option. When the existing literature was examined, this view was supported by the fact that a small number of molecular-based detection strategies have been developed, mainly targeting the $16 \mathrm{~S}$ rRNA genes, and which are applied with variable success.

The availability of the genome sequence would begin to provide a basis upon which to extend our knowledge of these bacteria and these data could be expected to support the development of improved diagnostic biomarkers. Determining the complete genome of one or more etiological agents of BPS would provide a suitable reference, from which to identify candidate gene(s) that could be assessed for their utility as diagnostic markers in the development of new PCR-based detection methods. As a step toward this objective, in this paper we report the whole genome sequence of C. estertheticum DSM 8809, an important etiological agent identified in episodes of BPS. 


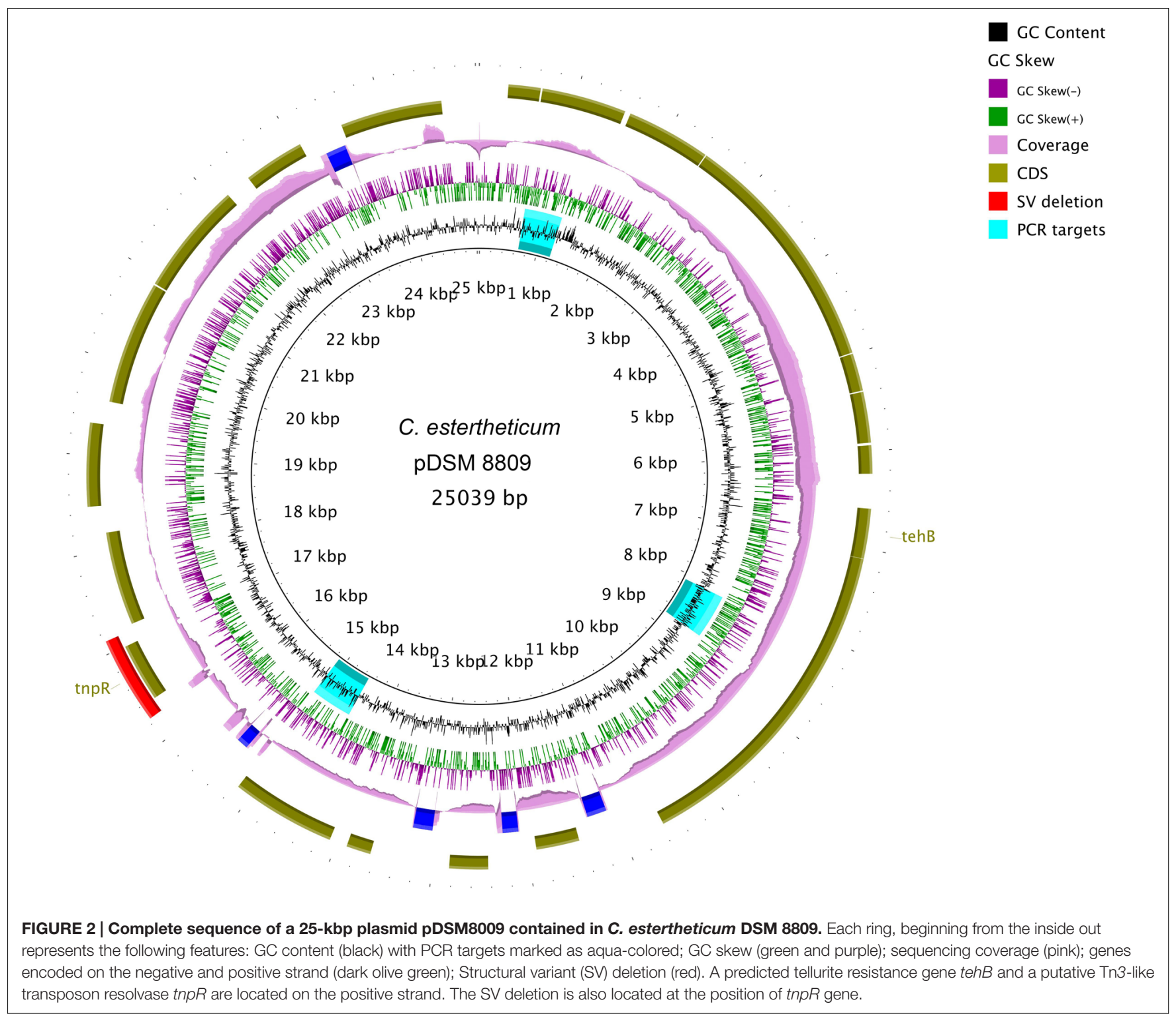

\section{MATERIALS AND METHODS}

\section{Bacterial Culture}

Clostridium estertheticum subsp. estertheticum DSM 8809 was acquired from Leibniz Institute DSMZ-German Collection of Microorganisms and Cell Cultures. The bacterium was grown in brain-heart infusion (BHI) broth under anaerobic conditions at $8^{\circ} \mathrm{C}$ for at least 2 weeks prior to the purification of genomic template DNA (gDNA).

\section{DNA Purification and Sequencing}

Genomic DNA was purified from C. estertheticum DSM 8809 using a phenol-chloroform method, that was modified from those previously reported and used with Clostridium difficile (Wren and Tabaqchali, 1987; Bouillaut et al., 2011). In brief, $5 \mathrm{ml}$ grown cultures of bacteria were harvested by centrifuging at $10,000 \times g$ for $2 \mathrm{~min}$. The resulting pellet was resuspended in $1 \mathrm{ml} \mathrm{TE}$ buffer $(10 \mathrm{mM}$ Tris- $\mathrm{HCl}$ and $1 \mathrm{mM}$ EDTA, at $\mathrm{pH}$ 8 ). It was then centrifuged again at $10,000 \times g$ for $2 \mathrm{~min}$, and the pellet re-suspended in $200 \mu \mathrm{l}$ genomic DNA solution (consisting of $34.23 \mathrm{~g}$ sucrose $/ 100 \mathrm{ml} \mathrm{TE}$, filter sterilized) to which $50 \mu \mathrm{l}$ freshly prepared lysozyme $(50 \mathrm{mg} / \mathrm{ml})$ was added. This mixture was incubated for $90 \mathrm{~min}$ at $37^{\circ} \mathrm{C}$. Following this step, $100 \mu \mathrm{l} 20 \%[\mathrm{w} / \mathrm{v}]$ sarkosyl and $15 \mu \mathrm{l}$ RNase A $(10 \mathrm{mg} / \mathrm{ml})$ was then added incubated for a further $30 \mathrm{~min}$ at $37^{\circ} \mathrm{C}$. Fifteen $\mu \mathrm{l}$ proteinase $\mathrm{K}(10 \mathrm{mg} / \mathrm{ml})$ was then added and the solution was incubated for another $30 \mathrm{~min}$ at $37^{\circ} \mathrm{C}$. In the final step of the purification protocol, TE buffer was then added to bring the final volume to $600 \mu \mathrm{l}$. The solution was then mixed with $600 \mu \mathrm{l}$ 25:24:1 phenol/chloroform/isoamyl alcohol and centrifuged at $17,000 \times g$ for $10 \mathrm{~min}$. and the resulting upper aqueous phase transferred to a clean microfuge tube. All of the above steps following the addition of $600 \mu \mathrm{l}$ 
A

\section{Clostridium novyi NT \\ Clostridium tetani E88}

Clostridium perfringens str. 13

Clostridium baratii str. Sullivan

Clostridium botulinum A str. Hall Clostridium botulinum A str. ATCC 3502 Clostridium acetobutylicum ATCC 824

Clostridium kluyveri DSM 555

Clostridium pasteurianum DSM 525

Clostridium estertheticum DSM 8809

Clostridium cellulovorans 743B

Clostridium beijerinckii NCIMB 8052

B

Clostridium botulinum A str. Hall Clostridium botulinum A str. ATCC 3502

Clostridium baratii str. Sullivan

Clostridium perfringens str. 13

Clostridium tetani $\mathrm{E} 88$

Clostridium novyi NT

Clostridium pasteurianum DSM 525

Clostridium beijerinckii NCIMB 8052

Clostridium acetobutylicum ATCC 824

Clostridium estertheticum DSM 8809

Clostridium cellulovorans 743B

Clostridium kluyveri DSM 555

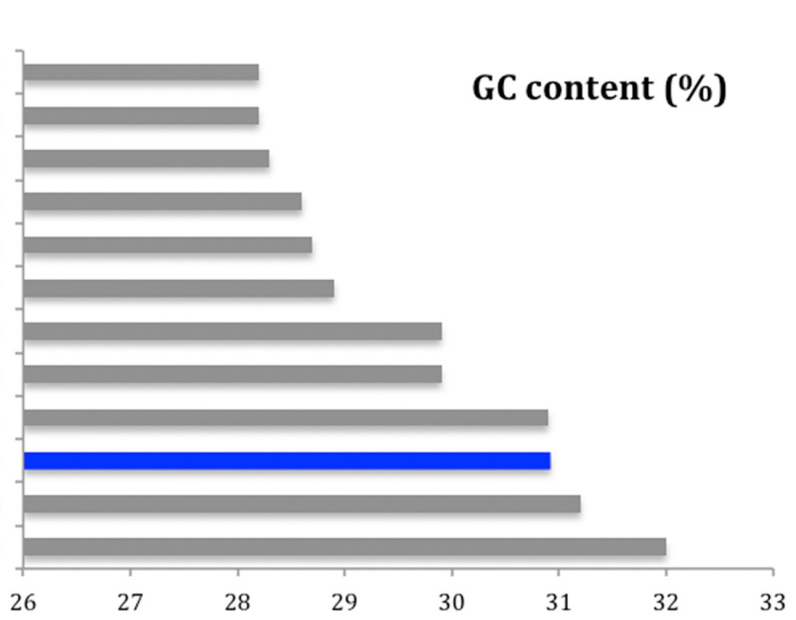

FIGURE 3 | Comparative analysis of genome size (A) and GC content (B) of C. estertheticum DSM 8809 with a selection of other available genomes representative of Clostridium species.

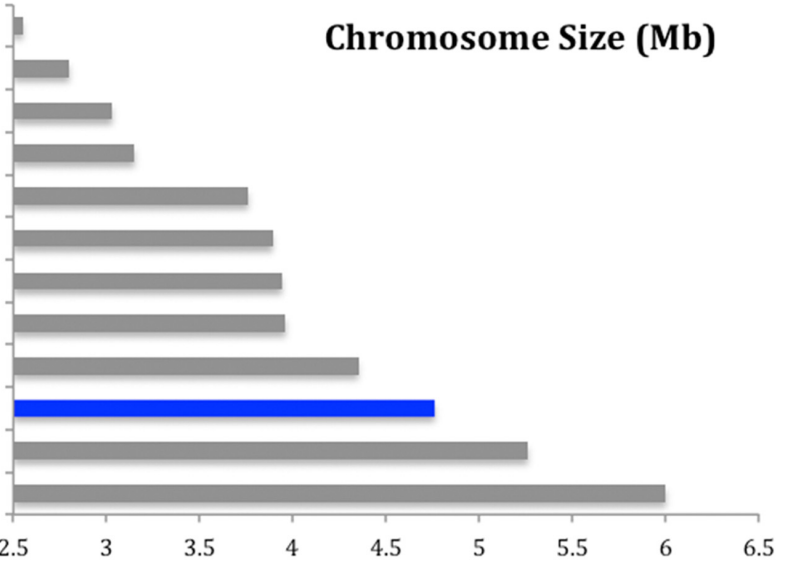

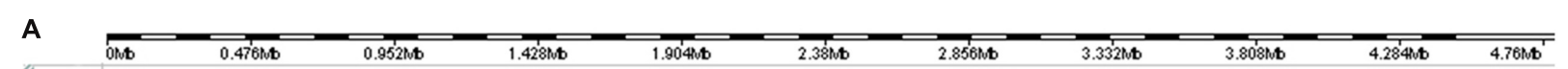

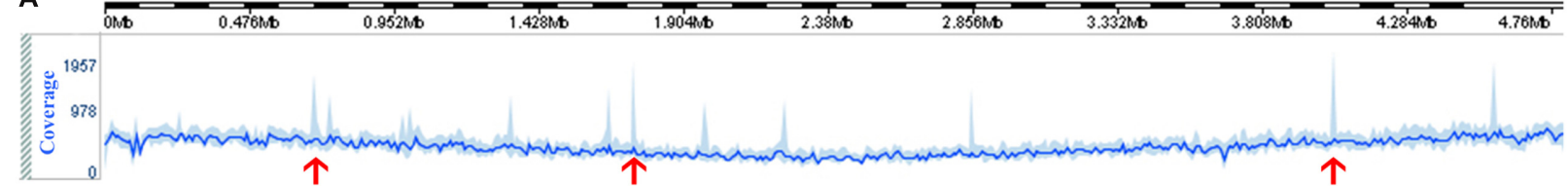

B

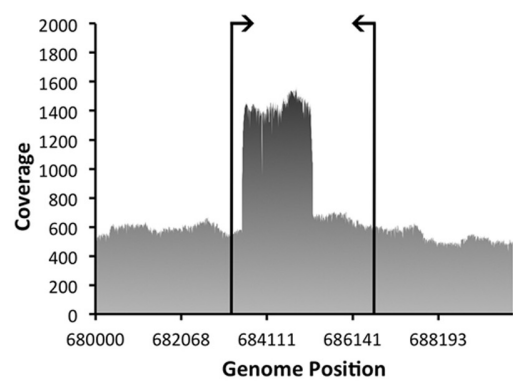

C

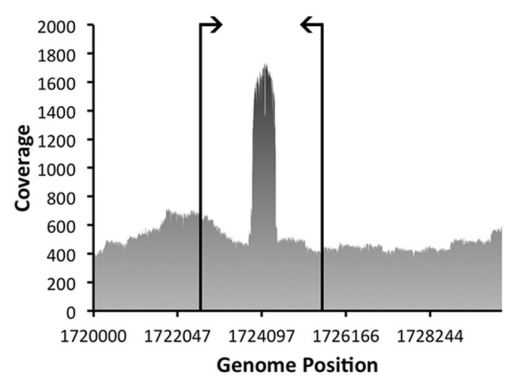

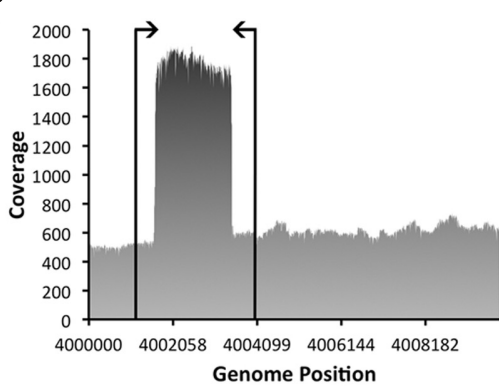

FIGURE 4 | Coverage information related to the assembly of the genome of C. estertheticum DSM 8809. The line in bright blue (A), located toward the top of the figure, represents the average sequence coverage of each window, denoted as (B-D) consisting of 10,000 bp and the area in lighter blue indicates the maximum and minimum coverage of the window. Spikes marked with red arrows highlight those regions referred to in the text and which were subsequently checked by PCR to validate the true nature of the consensus sequence. Annealing positions of the PCR primers are indicated by vertical lines, as shown for (B-D). 
TABLE 1 | Oligonucleotide primer sequences designed to validate the genomic sequence of Clostridium estertheticum DSM 8809.

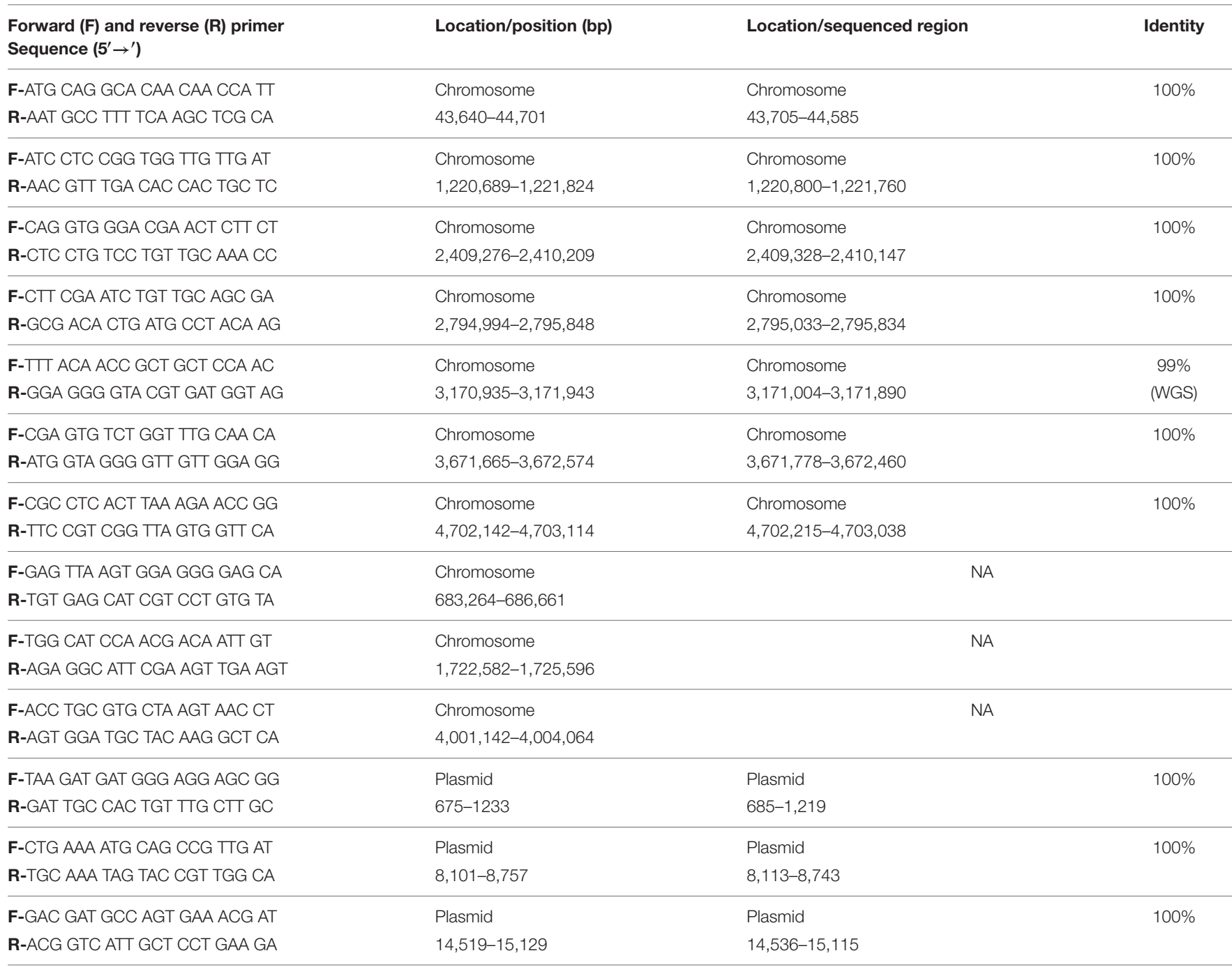

\section{PCR ATCTTTATCTTTTAaTAagtaAgCTAaAtaAGCAagtCCCAT-ACTA 11111111111111011011111111111111 WGS ATCTTMATCTTTTAATAAGTAAGCTAAATAAGCAAGTCCCATTACTA}

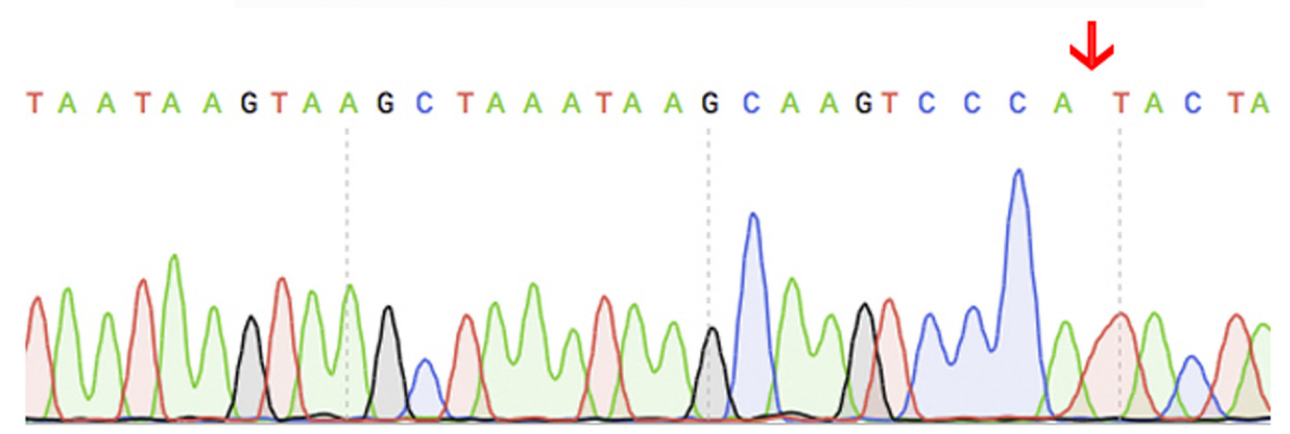

FIGURE 5 | A schematic showing the single nucleotide difference found between the sequence of PCR products and the whole genome sequencing data. This difference was caused by unclear sequence signal of the PCR product. These results indicate that the whole genome sequence is of high quality. 
TABLE 2 | General comparative analysis of C. estertheticum DSM 8809; Clostridium botulinum A str. Hall and Clostridium perfringenes str. 13.

\begin{tabular}{|c|c|c|c|}
\hline Features & C. estertheticum DSM 8809 & C. botulinum A str. Hall & C. perfringens str. 13 \\
\hline Genome size (bp) & $4,760,574$ & $3,760,560$ & $3,031,430$ \\
\hline No. of plasmids & 1 & 0 & 1 \\
\hline $\mathrm{G}+\mathrm{C}$ content $(\%)$ & 30.92 & 28.18 & 28.57 \\
\hline No. of predicted coding sequences (CDS) & 4,391 & 3,397 & 2,670 \\
\hline No. of rRNA operons & 47 & 24 & 30 \\
\hline No. of tRNA operons & 91 & 81 & 98 \\
\hline No. of ncRNA operons & 76 & 64 & 56 \\
\hline \multirow[t]{3}{*}{ No. of phages (PHAST) } & One intact & Three incomplete & One incomplete \\
\hline & One incomplete & & \\
\hline & One questionable & & \\
\hline No. of CRISPR regions & 0 & 4 & 0 \\
\hline
\end{tabular}

phenol/chloroform/isoamyl alcohol were repeated twice to achieve a more pure gDNA template for sequencing. Upon completion of the final step, gDNA was precipitated by adding $50 \mu l 3 \mathrm{M}$ sodium acetate ( $\mathrm{pH} 5.2)$ and three volumes of cold $95 \%[\mathrm{v} / \mathrm{v}]$ ethanol. This solution was maintained at $-20^{\circ} \mathrm{C}$ for at least $1 \mathrm{~h}$. After that, the solution was centrifuged at $17,000 \times g$ for $5 \mathrm{~min}$. The pellet was washed with $500 \mu \mathrm{l}$ $70 \%[\mathrm{v} / \mathrm{v}]$ ethanol, air-dried before being finally re-suspended in $100 \mu \mathrm{l}$ TE buffer and allowed to dissolve at room temperature overnight.

Library preparation using the above purified gDNA and the subsequent sequencing was carried out commercially by the Centre for Genomic Research at the University of Liverpool. In brief, gDNA was sheared to approximately 10-kbp and then sequenced on a Pacific Biosciences (PacBio) RS II instrument (P4/C2 chemistry) with two single molecule real-time (SMRT ${ }^{\circledR}$ ) cells. This approach provides for the sequencing of longer reads and is suitable when a complete genome sequence is required.

\section{Genome Assembly}

De novo assembly of the read data obtained was completed by $\mathrm{SMRT}^{\circledR}$ Analysis using the Hierarchical Genome Assembly Process (HGAP) 2.0 protocol (Chin et al., 2013). Minimum seed read length was set at 12,000 bp for PreAssembler v2. Expected genome size was set to $4,800,000$ bp [as indicated by an HGAP 3 trial run (data not shown)]. This approach resulted in two contigs (with mean QV score at 48.8 and 47.7, respectively) that were subsequently circularized by Circlator 0.16.0 (Hunt et al., 2015) using corrected sub-reads generated by HGAP 2 (with 6,000 minimum seed read length). The 'RS_Resequencing.1' protocol on SMRT ${ }^{\circledR}$ Analysis was then used to align raw reads to the circularized genome for an improved consensus.

\section{Validation of the Genome Structure by Selected PCR Sampling of the Chromosome and Plasmid}

The coverage information of the genome was visualized by SMRT $^{\circledR}$ View which provided data on the sequence at intervals of 10,000 bp. The coverage obtained after sequencing for each base pair was determined by using the 'genomecov' function in 'BEDtools' (Quinlan and Hall, 2010) a strategy that would indicate regions that may contain spikes, related to lack of sequence conformity. PCR primers were then designed with the aid of 'Primer3' (Koressaar and Remm, 2007; Untergasser et al., 2012) to sample randomly around the chromosome and plasmid and to support the consensus assembly. As before, all amplicons were sequenced by traditional Sanger sequencing protocols. The resulting sequences were then merged by 'Contig Assembly Program' (CAP) in BioEdit (Hall, 2011).

\section{Plasmid DNA Extraction}

Plasmid DNA was purified from C. estertheticum DSM 8809 using an alkali lysis method (Bimboim and Doly, 1979). The resulting DNA pellet obtained from $5 \mathrm{ml}$ bacterial culture was re-suspended in $200 \mu \mathrm{l}$ of a solution containing $50 \mathrm{mM}$ glucose, $10 \mathrm{mM}$ Tris-HCl pH8, $10 \mathrm{mM}$ EDTA, $10 \mathrm{mg} / \mathrm{ml}$ lysozyme and $10 \mathrm{mg} / \mathrm{mL}$ RNase A and incubated for $1 \mathrm{~h}$ at $37^{\circ} \mathrm{C}$. To this mixture was added $400 \mu \mathrm{l} 0.2 \%$ [w/v] $\mathrm{NaOH}$ and $1 \%$ [w/v] SDS. Threehundred $\mu \mathrm{l} 7.5 \mathrm{M}$ ammonium acetate and $300 \mu \mathrm{l}$ chloroform as then added and incubated on ice for $10 \mathrm{~min}$. The solution was centrifuged and the supernatant was transferred to a new tube containing $200 \mu \mathrm{l}$ chilled precipitation buffer (30\% PEG200, $1.5 \mathrm{M} \mathrm{NaCl}$ ) and incubated on ice for $15 \mathrm{~min}$. The purified plasmid DNA was recovered after centrifugation at 10,000 $\times g$ and the pellet was finally re-suspended in $50 \mu \mathrm{l} 1 \mathrm{x}$ TE buffer.

\section{Gene Identification Using BacMet/VFDB}

The raw assembly was submitted to Prokka (Seemann, 2014) to identify and annotate genes. The output was used to search for similar matches in BacMet (Pal et al., 2014) and virulence factor database (VFDB) (Chen et al., 2011) databases using BLASTP (Camacho et al., 2009). Only alignments with over 70\% coverage of the genes from these databases were included in the final annotation. These results were then filtered according to percentage of identity (threshold 50\%) and differences in gene length (50 bp).

\section{KO Mapping}

Metabolic pathways were reconstructed for each of C. estertheticum DSM 8809, Clostridium botulinum Hall A 
TABLE 3 | Antibiotic/biocide/metal resistance genes identified following comparison of the genome sequence of C. estertheticum DSM 8809 with content located in selected databases.

\begin{tabular}{|c|c|c|}
\hline Gene & Location & Resistant to \\
\hline alu & Chromosome & Aluminum (Al) \\
\hline $\operatorname{ars} B$ & Chromosome & Arsenic (As) \\
\hline $\operatorname{ars} D$ & Chromosome & Arsenic (As) \\
\hline $\operatorname{ars} R$ & Chromosome & Arsenic (As) \\
\hline bmr3 & Chromosome & Norfloxacin, puromycin, and tosufloxacin \\
\hline cadA & Chromosome & Cadmium (Cd), zinc (Zn) \\
\hline copA & Chromosome & Copper (Cu) \\
\hline corA & Chromosome & $\begin{array}{l}\text { Magnesium (Mg), cobalt (Co), nickel (Ni), } \\
\text { manganese (Mn) }\end{array}$ \\
\hline corC & Chromosome & Cobalt (Co), magnesium (Mg) \\
\hline $\operatorname{crcB}$ & Chromosome & Camphor \\
\hline csoR & Chromosome & Copper (Cu) \\
\hline cutC & Chromosome & Copper (Cu) \\
\hline dnak & Chromosome & Copper (Cu) \\
\hline$d r r A$ & Chromosome & Daunorubicin and doxorubicin \\
\hline fabK & Chromosome & Triclosan \\
\hline fieF & Chromosome & $\begin{array}{l}\text { Iron }(\mathrm{Fe}) \text {, zinc }(\mathrm{Zn}) \text {, cobalt }(\mathrm{Co}) \text {, cadmium }(\mathrm{Cd}) \text {, } \\
\text { nickel }(\mathrm{Ni})\end{array}$ \\
\hline $\operatorname{marR}$ & Chromosome & Chloramphenicol and tetracycline \\
\hline merR1 & Chromosome & Mercury (Hg) \\
\hline$m d t A$ & Chromosome & Deoxycholate and novobiocin \\
\hline$m d t B$ & Chromosome & Deoxycholate and novobiocin \\
\hline$m d t K$ & Chromosome & $\begin{array}{l}\text { Acriflavine, benzalkonium, chloramphenicol, } \\
\text { deoxycholate, doxorubicin, ethidium, } \\
\text { fluoroquinolones, fosfomycin } \\
\text { tetraphenylphosphonium ion (TPP), and } \\
\text { trimethoprim }\end{array}$ \\
\hline$m n t H$ & Chromosome & $\begin{array}{l}\text { Manganese (Mn), iron (Fe), cadmium }(\mathrm{Cd}) \text {, } \\
\text { cobalt (Co), zinc }(\mathrm{Zn})\end{array}$ \\
\hline \multirow[t]{2}{*}{$m n t P$} & Chromosome & $\begin{array}{l}\text { Manganese }(\mathrm{Mn}), \text { magnesium }(\mathrm{Mg}) \text {, methyl } \\
\text { viologen, hydrogen }\end{array}$ \\
\hline & & Peroxide $\left(\mathrm{H}_{2} \mathrm{O}_{2}\right)$ \\
\hline $\bmod B$ & Chromosome & Tungsten (W), molybdenum (Mo) \\
\hline norm & Chromosome & $\begin{array}{l}\text { Acriflavine, ciprofloxacin, daunomycin, } \\
\text { doxorubicin, ethidium bromide, kanamycin, } \\
\text { norfloxacin, ofloxacin, and streptomycin }\end{array}$ \\
\hline ohrR & Chromosome & Organic hydroperoxide \\
\hline pstB & Chromosome & Arsenic (As) \\
\hline qacA & Chromosome & $\begin{array}{l}\text { Antiseptic and disinfectant compounds, e.g., } \\
\text { intercalating dyes, diamidines, and quaternary } \\
\text { ammonium salts }\end{array}$ \\
\hline $\operatorname{ruvB}$ & Chromosome & $\begin{array}{l}\text { Cetylpridininium chloride (CPC), chromium (Cr), } \\
\text { dodine and 2-nitroimidazole }\end{array}$ \\
\hline stp & Chromosome & Spectinomycin and tetracycline \\
\hline sugE & Chromosome & Quaternary ammonium compound \\
\hline $\operatorname{tet}(\mathrm{O})$ & Chromosome & Tetracycline \\
\hline vanW & Chromosome & Vancomycin \\
\hline \multirow[t]{3}{*}{$v m r A$} & Chromosome & $\begin{array}{l}\text { 4,6-diamidino-2-phenylindole (DAPI), } \\
\text { acriflavine, Ethidium }\end{array}$ \\
\hline & & $\begin{array}{l}\text { Bromide, tetraphenylphosphonium (TPP), } \\
\text { quaternary ammonium }\end{array}$ \\
\hline & & Compounds (QACs) \\
\hline ziaA & Chromosome & Zinc $(\mathrm{Zn})$ \\
\hline zupt & Chromosome & $\begin{array}{l}\text { Zinc }(\mathrm{Zn}) \text {, iron (Fe), cobalt (Co), nickel (Ni), } \\
\text { copper (Cu), Cadmium (Cd) }\end{array}$ \\
\hline $\operatorname{teh} B$ & Plasmid & Tellurite \\
\hline
\end{tabular}

and Clostridium perfringens 13 using ORFs as identified by RAST annotation (Aziz et al., 2008) and the KEGG Automatic Annotation Server (Kanehisa and Goto, 2000). Amino acid sequences for each ORF were provided and orthologs were assigned using the bi-directional best hit (BBH) method. The representative gene data set "for Prokaryotes" was used in addition to the inclusion of manually annotated organisms C. botulinum Hall A (cbh) and C. perfringens 13 (cpe) for the mapping of all three isolates.

\section{RESULTS}

\section{Whole Genome Sequencing of Clostridium estertheticum DSM 8809}

Several DNA extraction protocols reported by previous researchers were assessed for their suitability to provide purified gDNA of sufficient concentration and quality for SMRT ${ }^{\circledast}$ sequencing. Following several attempts the phenol-chloroform method was considered to be suitable and capable of yielding higher qualities of gDNA that would meet the $\mathrm{SMRT}^{\circledR}$ sequencing requirements (data not shown). This enabled the purification of a good quality sequencing template that resulted in 175,417 reads and 3,526,834,644 bases being produced following SMRT ${ }^{\circledR}$ sequencing.

De novo assembly with HGAP3 using the default settings produced 11 polished contigs (data not shown). After optimizing of the assembly protocol two contigs were produced, that constituted the complete genome sequence of C. estertheticum DSM 8809 (GenBank accession number CP015756 and CP015757). The genome consisted of two circularized DNA molecules that included, a 4,760,574 bp chromosome (Figure 1) and a 25,039 bp plasmid pDSM8809 (Figure 2). Figure 3 provides a comparison of the general features of the genome of C. estertheticum DSM 8809 with other species of this genus. The chromosome of the latter is one of the largest in the genus (Figure 3A) whilst its GC content of $30.92 \%$, was lower than C. cellulovorans and C. kluyveri, both of which are at the upper end of the range (Figure 3B).

The quality of these data was then assessed. Based on this evaluation, the genome has a mean coverage of 522.14-fold. Analyses of the coverage graph (Figure 4) highlighted a number of spikes that reflected areas with significantly higher coverage. These spikes may have resulted from the merging of repeat regions contained within the genome. In order to validate the genome sequence, three sets of PCR primers were designed, each flanking a coverage spike (see Table 1; Figure 4). The size of the amplicons obtained after PCR were in complete agreement with the predicted size from the genome, confirming the accuracy of the assembly (data not shown).

An additional set of seven oligonucleotide primers were designed to target random positions across the circular chromosome together with a further three located on the plasmid (Table 1). All amplicons were successfully aligned to the genome with $100 \%$ identity. One of these contigs demonstrated a single nucleotide polymorphism (Figure 5). The raw sequencing data showed that this was most likely caused by an unclear 


\section{Clostridium estertheticum DSM 8809}

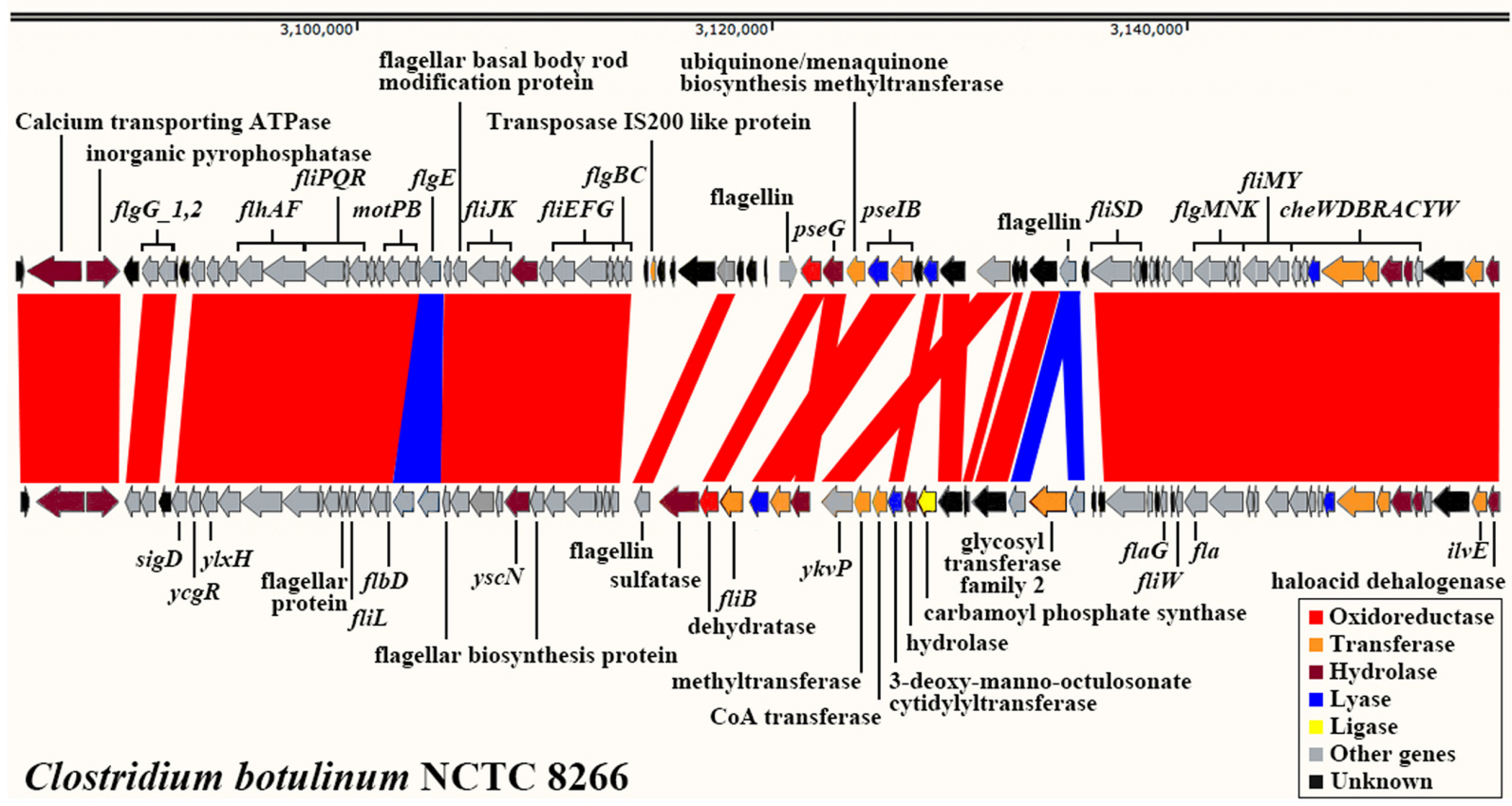

FIGURE 6 | A representation showing the alignment of flagella-related gene clusters comparing those identified in C. estertheticum DSM 8809 with similar loci in Clostridium botulinum NCTC 8266. Each arrow shown represents for a single CDS.

sequencing signal from the amplicons produced. These validation checks supported the quality of the assembled genome for C. estertheticum DSM 8809.

During genome annotation, a total of 4,391 CDS were predicted along with 91 tRNAs, 47 rRNAs, 76 ncRNAs and a further 24 CDS were identified on the plasmid pDSM8809. No CRISPR sequences were identified in the C. estertheticum DSM 8809 genome, by Prokka. Nonetheless a 109-bp putative CRISPR was located using CRISPRfinder (Grissa et al., 2007) on the chromosome at position 803654-803763, wherein a hypothetical protein coding sequence was identified at this locus (Table 2).

To assess the metabolic potential of C. estertheticum DSM 8809, draft metabolic networks were generated using ORFs predicted by RAST annotation and KEGG in comparison with two other Clostridium species, namely botulinum str. Hall and perfringens str. 13 (Table 2). In C. estertheticum DSM 8809, a total of 2,003 of 4,504 ORFs were mapped to KEGG pathways. This number was higher than what those observed for $C$. botulinum str. Hall (1,608 mapped genes) or C. perfringens str. 13 (1,385 mapped genes) and which also mapped to KEGG. Among these, enzymes required to utilize various carbon-based sources including arabinose, cellobiose, galactose, inositol, maltose, mannose, melibiose, raffinose, rhamnose, ribose, salicin, starch, and sucrose were identified. This finding broadly supports the biochemical profiling of C. estertheticum DSM 8809, as reported earlier by Spring et al. (2003) and highlights the saccharoltyic capacity of this bacterium. Further, enzymes identified in C. estertheticum DSM 8809 and that were mapped to KEGG, and which are required for utilizing arabinose, cellobiose, maltose and rhamnose, were absent in either C. botulinum str. Hall or C. perfringens str. 13 .

When considering the further metabolism of pyruvate, pathways required for its biochemical transformation resulting in the production of non-gaseous fermentation products including butyrate, acetate, lactate, and others were also identified.

\section{Antibiotic Genes Annotated in the Genome}

No acquired antimicrobial resistance genes were identified by ResFinder 2.1 (Zankari et al., 2012). Prokka predicted a number of genes that encoded resistance to several antimicrobial compounds, including biocides and these are summarized in Table 3. Additional biocide and metal resistance genes were predicted using BacMet (Pal et al., 2014) and these results are also included in Table 3. Three putative prophage regions were predicted by PHAST (Zhou et al., 2011), among which a $31.5 \mathrm{kbp}$ region $(3,000,409-3,031,979)$ and a $19.1 \mathrm{kbp}(3,034,721-$ $3,053,909)$ region were located very close to each other. The third prophage region of $59.2 \mathrm{kbp}$ in length $(3,504,461-3,563,662)$ identified contained a predicted transposase gene from Tn916, together with a predicted cusS gene related to copper $(\mathrm{Cu})$ and silver (Ag) resistance.

Structural variations (SV) within the raw sequence reads across the genome were identified using Sniffles $0.1 .0^{1}$. In summary 81 deletions, 4 duplications, 588 insertions, and 43 inversions were found in the chromosome, while 1 deletion,

\footnotetext{
${ }^{1}$ https://github.com/fritzsedlazeck/Sniffles
} 
1 duplication, 13 insertions, and no inversion were found in the plasmid.

The 23,034-bp plasmid pDSM8809 was mainly composed of genes of unknown function. Those that could be identified included a predicted transposon Tn3-like resolvase-encoding $\operatorname{tnpR}$. Interestingly, one of the deletions identified by SV analysis included the $\operatorname{tn} p R$ gene (see Figure 2). The plasmid was also predicted to carry a teh $B$ gene that is known to contribute to tellurite resistance.

\section{Virulence Genes Annotated in the Genome Sequence}

Clostridium estertheticum is not known to be pathogenic to human health. To determine whether or not any virulence factors may be present, the genome was searched using BLASTP and the virulence factor database (VFDB) (Chen et al., 2011).

Based on this output 54 potential virulence factors were identified in the genome. For example, several flagella-related genes were identified and located on the C. estertheticum DSM 8809 genome. A similar cluster was also identified in C. botulinum NCTC 8266 (Accession number: NZ_CP010520) with a number of notable differences and illustrated in Figure 6. In particular, it was noted that there was an area where the genes have been reordered and which was flanked by two conserved loci. Most genes in this particular location are coded on the negative strand. The less conserved region consisted of genes coding for flagellin, a component of bacterial flagella, and included the pseGIB genes whose function is to modify flagellin. C. estertheticum DSM 8809 also possessed an insertion sequence denoted as IS200 and which was absent from the genome of C. botulinum NCTC 8266. In contrast, the fliB gene identified in the latter bacterium was not found in C. estertheticum DSM 8809. Genes upstream and downstream of this region were found to be more conserved between these organisms. As an example of this, a group of flagellar related genes including flgBC, fliEFGJKLPQR, $f l b D, \operatorname{mot} P B, f l h A F$ were located upstream whilst fliSDMY, flaG, cheWDBRACYW located downstream.

PathogenFinder 1.1 (Cosentino et al., 2013), a program that runs comparisons between pathogenic and non-pathogenic bacteria using whole genome sequence data, showed that, when C. estertheticum DSM 8809 was used as the query sequence, results demonstrated that it was non-pathogenic with an accuracy of $88.6 \%$.

\section{DISCUSSION}

The complete genome sequence of C. estertheticum DSM 8809 was determined. Following SMRT ${ }^{\circledast}$ sequencing, the genome was assembled, into a single contig of $4,760,574$ bp representing

\section{REFERENCES}

Aziz, R. K., Bartels, D., Best, A. A., DeJongh, M., Disz, T., Edwards, R. A., et al. (2008). The RAST server: rapid annotations using subsystems technology. BMC Genomics 9:75. doi: 10.1186/14712164-9-75 the bacterial chromosome along with a smaller contig of 25,039 bp representing a plasmid pDSM8809. Compared with other members of this bacterial genus such as C. botulinum and C. perfringens, C. estertheticum harbors a larger genome with a marginally higher GC content.

Enzymes that contributed to the metabolism of carbonsources supporting the saccharolytic phenotype of this bacterium were identified. Furthermore, pathways contributing to endproducts of anaerobic metabolism, including butyrate, acetate, and lactate among others were noted. These observations support earlier work describing the biochemical profile of C. estertheticum DSM 8809.

The genome was queried in an effort to identify genes potentially important to public health, namely antibiotic/biocide/metal resistance-encoding genes and virulence factors based on sequence similarity. Our analysis identified a flagellar-related gene cluster, multiple genes potentially related to antibiotic, biocide and metal resistance, along with several predicted virulence factor genes, and a transposase-encoding gene on the plasmid. Besides the findings above, the high quality complete genome sequence generated in this study also forms an important basis for further studies that will lead to a better understanding and control of BPS. Use of the genes annotated in this genome may prove useful for the selection of candidate diagnostic markers to be assessed for inclusion in molecularbased assays designed to facilitate the rapid discovery of the bacterium on vacuum-packed meat surfaces, thereby improving food quality and reducing economic loss.

\section{AUTHOR CONTRIBUTIONS}

ZY and SF designed the study. ZY, LG, EB, and RR participated in the lab work. ZY conducted the bioinformatics analysis with the help and supervising from PG and DH. PW, DB, and SF supervised the whole project.

\section{FUNDING}

The funding for this research was provided by the Food Institutional Research Measure (FIRM) administered by the Department of Agriculture, Food and the Marine (Ireland).

\section{ACKNOWLEDGMENT}

We thank our colleagues at the UCD School of Veterinary Medicine for providing facilities to enable us to culture the bacterium.

Bimboim, H. C., and Doly, J. (1979). A rapid alkaline extraction procedure for screening recombinant plasmid DNA. Nucleic Acids Res. 7, 1513-1523. doi: 10.1093/nar/7.6.1513

Bolton, D. J., Walsh, D., and Carroll, J. (2015). A four year survey of blown pack spoilage Clostridium estertheticum and Clostridium gasigenes on beef primals. Lett. Appl. Microbiol. 61, 153-157. doi: 10.1111/lam.12431 
Bouillaut, L., McBride, S. M., and Sorg, J. A. (2011). Genetic manipulation of Clostridium difficile. Curr. Protoc. Microbiol. Chap. 9, Unit 9A.2. doi: 10.1002/9780471729259.mc09a02s20

Broda, D. M., Delacy, K. M., Bell, R. G., Braggins, T. J., and Cook, R. L. (1996). Psychrotrophic Clostridium spp. associated with 'blown pack' spoilage of chilled vacuum-packed red meats and dog rolls in gas-impermeable plastic casings. Int J. Food Microbiol. 29, 335-352. doi: 10.1016/0168-1605(95)00070-4

Camacho, C., Coulouris, G., Avagyan, V., Ma, N., Papadopoulos, J., Bealer, K., et al. (2009). BLAST+: architecture and applications. BMC Bioinformatics 10:421. doi: 10.1186/1471-2105-10-421

Chen, L., Xiong, Z., Sun, L., Yang, J., and Jin, Q. (2011). VFDB 2012 update: toward the genetic diversity and molecular evolution of bacterial virulence factors. Nucleic Acids Res. 40, D641-D645.

Chin, C. S., Alexander, D. H., Marks, P., Klammer, A. A., Drake, J., Heiner, C., et al. (2013). Nonhybrid, finished microbial genome assemblies from long-read SMRT sequencing data. Nat. Methods 10, 563-569. doi: 10.1038/nmeth.2474

Cosentino, S., Larsen, M. V., Aarestrup, F. M., and Lund, O. (2013). PathogenFinder-Distinguishing friend from foe using bacterial whole genome sequence data. PLoS ONE 8:e77302. 10.1371/journal.pone.0077302

Grissa, I., Vergnaud, G., and Pourcel, C. (2007). CRISPRFinder: a web tool to identify clustered regularly interspaced short palindromic repeats. Nucleic Acids Res. 35, W52-W57.

Hall, T. (2011). BioEdit: an important software for molecular biology. GERF Bull. Biosci. 2, 60-61.

Hunt, M., De Silva, N., Otto, T. D., Parkhill, J., Keane, J. A., and Harris, S. R. (2015). Circlator: automated circularization of genome assemblies using long sequencing reads. Genome Biol. 16, 1-10. doi: 10.1186/s13059-015-0849-0

Kanehisa, M., and Goto, S. (2000). KEGG: kyoto encyclopedia of genes and genomes. Nucleic Acids Res. 28, 27-30. doi: 10.1093/nar/28.1.27

Koressaar, T., and Remm, M. (2007). Enhancements and modifications of primer design program Primer3. Bioinformatics 23, 1289-1291. doi: 10.1093/bioinformatics/btm091

Pal, C., Bengtsson-Palme, J., Rensing, C., Kristiansson, E., and Larsson, D. J. (2014). BacMet: antibacterial biocide and metal resistance genes database. Nucleic Acids Res. 42, D737-D743. doi: 10.1093/nar/gkt1252
Quinlan, A. R., and Hall, I. M. (2010). BEDTools: a flexible suite of utilities for comparing genomic features. Bioinformatics 26, 841-842. doi: 10.1093/bioinformatics/btq033

Seemann, T. (2014). Prokka: rapid prokaryotic genome annotation. Bioinformatics 30, 2068-2069.

Spring, S., Merkhoffer, B., Weiss, N., Kroppenstedt, R. M., Hippe, H., and Stackebrandt, E. (2003). Characterization of novel psychrophilic clostridia from an Antarctic microbial mat: description of Clostridium frigoris sp. nov., Clostridium lacusfryxellense sp. nov., Clostridium bowmanii sp. nov. and Clostridium psychrophilum sp. nov. and reclassification of Clostridium laramiense as Clostridium estertheticum subsp. laramiense subsp. nov. Int. J. Syst. Evol. Microbiol. 53, 1019-1029.

Untergasser, A., Cutcutache, I., Koressaar, T., Ye, J., Faircloth, B. C., Remm, M., et al. (2012). Primer3-new capabilities and interfaces. Nucleic Acids Res. 40, e115-e115. doi: 10.1093/nar/gks596

Wren, B. W., and Tabaqchali, S. O. A. D. (1987). Restriction endonuclease DNA analysis of Clostridium difficile. J. Clin. Microbiol. 25, 2402-2404.

Zankari, E., Hasman, H., Cosentino, S., Vestergaard, M., Rasmussen, S., Lund, O., et al. (2012). Identification of acquired antimicrobial resistance genes. J. Antimicrob. Chemother. 67, 2640-2644. doi: 10.1093/jac/ dks261

Zhou, Y., Liang, Y., Lynch, K. H., Dennis, J. J., and Wishart, D. S. (2011). PHAST: a fast phage search tool. Nucleic Acids Res. 39, W347-W352.

Conflict of Interest Statement: The authors declare that the research was conducted in the absence of any commercial or financial relationships that could be construed as a potential conflict of interest.

Copyright (c) 2016 Yu, Gunn, Brennan, Reid, Wall, Gaora, Hurley, Bolton and Fanning. This is an open-access article distributed under the terms of the Creative Commons Attribution License (CC BY). The use, distribution or reproduction in other forums is permitted, provided the original author(s) or licensor are credited and that the original publication in this journal is cited, in accordance with accepted academic practice. No use, distribution or reproduction is permitted which does not comply with these terms. 